\title{
Primary Structure and Organ-Specific Expression of the Rat Aryl Hydrocarbon Receptor Repressor Gene
}

\author{
Hiroko Nishinashi, Yuichiro Kanno, Kaori Tomuro, Takayuki Nakahama, and Yoshio Inouye* \\ Faculty of Pharmaceutical Sciences, Toho University; 2-2-1 Miyama, Funabashi, Chiba 274-8510, Japan. \\ Received October 1, 2005; accepted December 29, 2005
}

The aryl hydrocarbon receptor repressor (AhRR) is a member of the basic helix-loop-helix/Per-Arnt-Sim (bHLH/PAS) family of transcription factors, providing a negative feedback loop with a xenobiotic or endogenous ligand-dependent signal transduction mediated by the AhR. We sequenced full-length AhRR mRNA extracted from the heart of a male Wistar rat injected intraperitoneally with 3-methylcholanthrene (3-MC) $24 \mathrm{~h}$ before. The $95.6 \mathrm{~kb}$-long AhRR genome was clarified to consist of 11 exons and 10 introns. The constitutive expression of AhRR mRNA was prominent in males when compared with females in parallel with the sexual difference in AhR expression. Although AhRR was ubiquitously expressed in all tissues tested, the levels of AhRR expression were higher in the small intestine, where the 3-MC-dependent induction of CYP1A1 transcription was less significant, than in the heart, lung, liver, and kidney. The dose-dependent suppression of AhR-dependent transcriptional activation in both the presence and absence of 3-MC was observed in rat liver-derived RL-34 cells transiently transfected with the expression plasmid for AhRR in combination with the reporter plasmid.

Key words arylhydrocarbon receptor repressor; full-length mRNA sequence; tissue-specific expression

The aryl hydrocarbon receptor repressor (AhRR) was discovered as a protein that negatively regulates the function of AhR, by which the 3-methylcholanthrene (3-MC)-dependent induction of the transcription of AhRR is mediated. ${ }^{1,2)}$ AhRR belongs to the Per-Arnt-Sim (PAS) family of the basic helixloop-helix (bHLH) superfamily along with AhR and ARNT. The bHLH domain, leucine zipper, and PAS domain are commonly shared by bHLH/PAS family proteins. The bHLH domain takes part in the binding to DNA and formation of heterodimer or homodimer, while the leucine zipper and PAS domain participate in the recognition of the dimer partner when heterodimer or homodimer is formed. Moreover, the PAS domain usually consists of PAS A and PAS B and has functions such as the interaction with ligand and HSP90, especially in the PAS B domain. ${ }^{2}$ Since the Q (glutamine)-rich domain, which is the transcriptional activation domain, and PAS B domain are missing, AhRR is considered to be defective in both ligand and HSP90 binding and transcriptional activation, although the structure of AhRR is similar to that of AhR. ${ }^{3)}$ An experiment using mice showed the nuclear localization of AhRR even in the absence of any ligand in marked contrast to AhR, which forms a cytoplasmic complex in its latent state. ${ }^{4)}$ The competition with AhR for the common heterodimer partner ARNT, and the occupation by the AhRRARNT complex of the AhR-responsive element (AhRE) without transactivation of target genes might be responsible for the inhibitory effect of AhRR on AhR-mediated gene expression. The physiologic role of AhRR is thought to be as a functional modifier of AhR due to the existence of AhRE arrays, which are responsible for the AhR-dependent transcriptional activation upstream from the AhRR gene. Although the true function of AhRR remains to be elucidated, AhRR orthologues have been reported in mice, ${ }^{1,5}$ monkeys, and fish. ${ }^{6)}$ Recently, duplicate AhRR genes, AhRR1 and AhRR2, were discovered for the first time in zebra fish. ${ }^{7)}$

While this study was in progress, the sequence of rat AhRR cDNA was reported (accession number AY367561), although $3^{\prime}$-UTR was only partially sequenced. ${ }^{3)}$ In this re- search, the full-length cDNA of rat AhRR was sequenced (deposited with accession number AB174900) to clarify the so far unidentified genomic structure and compare the mRNA structure with those of other species highly divergent in $3^{\prime}$-UTR. ${ }^{5,8)}$ Next, the constitutive expression of the AhRR transcript and the responsiveness to AhR-mediated induction were studied in parallel with those of other bHLH/PAS proteins, i.e., AhR, ARNT, and ARNT2, and AhR-targeted CYP1A1, by reverse transcriptional PCR in various organs such as the liver, kidney, heart, lung, cecum, and longitudinally fragmented small intestine. ${ }^{9-10)}$ The AhRR-expression plasmid was constructed to evaluate the functional behavior of AhRR in rat liver-derived RL-34 cells.

\section{MATERIALS AND METHODS}

Animals and Treatment Seven-week-old male and female Wistar rats (Clea, Japan) were kept under a 12-h lightdark cycle and provided with food and water ad libitum. The rats were killed and the organs dissected $24 \mathrm{~h}$ after treatment with $3-\mathrm{MC} 80 \mathrm{mg} / \mathrm{kg}$ body weight in corn oil.

Amplification of an Internal Fragment of AhRR cDNA by Reverse-Transcriptional PCR Total RNA was extracted from the homogenate of $c a .90 \mathrm{mg}$ of dissected organs using the RNeasy Mini Kit (Qiagen, Hilden, Germany). To prevent RNA hydrolysis, all procedures were conducted on ice. The total RNA extract thus prepared and oligo-dT primer were added to RTG You-Prime First-Strand Beads (Amersham Biosciences, Piscataway NJ, U.S.A.). Reverse transcription proceeded at $37^{\circ} \mathrm{C}$ for $1 \mathrm{~h}$ to obtain cDNA, to which the primer pair, Pyrobest DNA polymerase (TAKARA, Kyoto, Japan), 10× Pyrobest Buffer II, and dNTP mixture were added. After adjusting the total volume to $25 \mu \mathrm{l}$, cDNA was amplified by 30 cycles of denaturation at $95^{\circ} \mathrm{C}$ for $45 \mathrm{~s}$, annealing at $58^{\circ} \mathrm{C}$ for $45 \mathrm{~s}$, and extension at $72^{\circ} \mathrm{C}$ for $5 \mathrm{~min}$ in a thermal cycler. The following oligonucleotides designed by consulting with the reported cDNA sequence of the mouse AhRR were used as sense and antisense 
primer pairs: 5'-GGCTTACCATGGGAACTGAG-3' (bases 147-166) and 5'-GCAGCAACTCTAGGGTAGGAAAAT3' (reverse complement of bases 2171-2194) for coding sequence; and 5'-CAGAGCCTCCTCATCACCTC-3' (bases 1971-1990) and 5'-GCAGCAACTCTAGGGTAGGAAAAT-3' (bases 4301-4320) for 3'-UTR.

Subcloning of PCR Products The PCR-amplified cardiac AhRR cDNAs were recovered from agarose gel using the DNA Fragment Purification Kit MagExtractor (Toyobo) and ligated to a pCR 4Blunt-TOPO vector (Invitrogen Corp.). Escherichia coli competent cells (XL-1 Blue) transformed with $100 \mathrm{ng}-1 \mu \mathrm{g}$ of plasmid DNA were plated on Luria-Broth (LB)/Amp plates and incubated at $37^{\circ} \mathrm{C}$ overnight. White single colonies were selected from the plates and incubated in LB supplemented with ampicillin and tetracycline overnight. Plasmids harboring the RT-PCR products were purified using the DNA Fragment Purification Kit MagExtractor (Toyobo).

5'-RACE and 3'-RACE The untranslated regions of cardial AhRR mRNA were sequenced using the GeneRacer Race-ready cDNA kit (Invitrogen). Total RNA was extracted from rat hearts, injected intraperitoneally with 3-MC $24 \mathrm{~h}$ previously. Primers for 5' - and $3^{\prime}$-RACE analysis were:

The initial PCR for the $5^{\prime}$-end utilized GeneRacer 5' primer and 5'-CCACCAGAGCAAAGCCATTGAG-3' as a gene-specific primer (reverse complement of bases 428449). Nested PCR utilized GeneRacer 5'-nested primer and 5'-CTCAGTTCCCATGGTAAGCC-3' as a gene-specific nested primer (reverse complement of bases 147-165). The initial PCR for the 3 '-end utilized 5'-TGCCCTAGTGAAGCCCATA-3' as a gene-specific primer (bases 4039-4055) and GeneRacer reverse 3'-primer. Nested PCR utilized 5'-GCAGGTCAGGCTTTGTGGAC-3' as a gene-specific primer (bases 4160 - 4179) and GeneRacer 3'-nested primer.

Sequencing The nucleotide sequencing of PCR products was carried out using the Thermo Sequenase Cy5.5 Dye Terminator Cycle Sequencing Kit (Amersham Biosciences) and GeneRapid (Amersham Biosciences, Model Seq4×4). Vector-specific sequence primers were as follows with the annealing temperatures in parentheses: T3 HT sense primer $\left(54^{\circ} \mathrm{C}\right), 5^{\prime}$-AATTAACCCTCACTAAAGGGAAC- $3^{\prime}$; and T7 HT antisense primer $\left(58^{\circ} \mathrm{C}\right), 5^{\prime}$-GTAATACGACTCACTATAGGGCGA-3'.

Gene Structure The structure of the rat Ahrr gene was determined using the BLAST program and the reported genomic database. Exon boundaries were inferred through comparison of the cDNA sequence determined in this study with the genomic database sequence.

Rat AhRR Expression Construct and Reporter Plasmid Total RNA extract and oligo-dT primer were added to RTG You-Prime First-Strand Beads (Amersham Biosciences). After leaving at room temperature for $1 \mathrm{~min}$, reverse transcription proceeded at $37^{\circ} \mathrm{C}$ for $1 \mathrm{~h}$ to obtain cDNA, to which the primer pair, Pyrobest DNA polymerase (TAKARA, Japan), 10× Pyrobest Buffer II, and dNTP mixture were added. The PCR utilized 5'-ATGATGATTCCGTCAGGAGA-3' as a sense primer (bases 80 - 99, start codon underlined) and 5'-CTAGGGTAGGAAAATCCCAT$3^{\prime}$ as an antisense primer (reverse complement of bases 2166 - 2185, stop codon underlined) with the following PCR parameters: $95^{\circ} \mathrm{C}$ for $5 \mathrm{~min}$ followed by 50 cycles at $98^{\circ} \mathrm{C}$ for $30 \mathrm{~s}$ (denaturation), $50^{\circ} \mathrm{C}$ for $30 \mathrm{~s}$ (annealing), and $72{ }^{\circ} \mathrm{C}$ for $3 \mathrm{~min}$ (extension) in a thermal cycler. The PCR-amplified AhRR cDNA was recovered from agarose gel using the DNA Fragment Purification Kit MagExtractor (Toyobo) and ligated to the EcoRV site of the pcDNA 5/TO vector after treatment with the Blunting Kination Ligation Kit (TaKaRa) to blunt and dephosphorylate their ends. pcDNA-AhRR plasmid harboring the RT-PCR product was purified using the GFX Micro Plasmid Prep Kit (AmershamBiosciences). The AhRE-pGL3- Promoter plasmid was prepared by cloning the AhRE sequence into the BglII site of the pGL3-Promoter vector (Promega, U.S.A.). The double-stranded AhRE nucleotide sequence was prepared by annealing the following synthetic oligomers: 5'-GATCTTGCGTGACAAGCCTTGCGTGAC AAGCTTGCGTGACAAGCTTGCGTGAC-3' and 5'-GATCGTCACGCAAGCTTGTCACGC AAGCTTGTCACGCAAGGCTTGTCACGCAA-3'. ${ }^{11-13)}$

Cell Culture, Transient Transfection, and Reporter Gene Assay Rat liver-derived RL-34 cells were maintained in Dulbecco's MEM (Sigma) supplemented with 10\% fetal calf serum at $37^{\circ} \mathrm{C}$ under $5 \% \mathrm{CO}_{2}$. RL-34 cells were subcultured on 24-well plates. Twenty-four hours later, the cells were transfected in each well with $200 \mathrm{ng}$ of AhRE-pGL3Promoter plasmid, $25 \mathrm{ng}$ of phRL-SV40 control plasmid (Promega), and pcDNA-AhRR plasmid (0 to $200 \mathrm{ng}$ ). In a negative control, pGL3-Promoter was employed instead of AhRE-pGL3-Promoter in the presence or absence of pcDNA-AhRR. The total DNA content was adjusted to $400 \mathrm{ng}$ with pcDNA empty plasmid. Cells were cultured overnight and treated with either $3-\mathrm{MC} 2.5 \mu \mathrm{M}$ or vehicle (DMSO) for the final $6 \mathrm{~h}$ before the reporter assay was performed using the Dual-Luciferase Reporter Assay System (Promega) and a Turner Designs Luminometer (Model TD20/20, Promega). The final luminescence values were expressed as the ratio of the firefly luciferase units to the $R e$ nilla luciferase units.

Construction and Function of Reporter Plasmid Inserted with Various AhRR Promoter Fragments Upstream the Firefly Luciferase Gene Genomic DNA was extracted from the homogenate of $c a .15 \mathrm{mg}$ of dissected liver using Genomic Prep Cells and Tissue DNA Isolation Kit (Amersham Biosciences). A DNA fragment of the promoter region of the rat AhRR genome was amplified with PCR and cloned into the pGL3-Basic vector. The AhRR $(-1221 /+71$, where +1 is the transcriptional start) DNA was amplified by 30 cycles of denaturation at $98^{\circ} \mathrm{C}$ for $30 \mathrm{~s}$, annealing at $69^{\circ} \mathrm{C}$ for $30 \mathrm{~s}$, and extension at $72^{\circ} \mathrm{C}$ for $3 \mathrm{~min}$ using 5'-AGAGTTCCTAGGGCGGGCTG-3' and 5'-CTGGGGCAGGCGGGGCGCAGAA-3' as sense and antisense primers, respectively, and cloned into the blunt-ended SmaI site of pGL3-Basic vector to give a novel plasmid AhRR $(-1221 /+71)$-pGL3-Basic. Likewise, AhRR $(-633 /+71)$, AhRR $(-269 /+71)$, AhRR $(-20 /+71)$, and AhRR $(-296 /$ -86) were PCR amplified with some modifications in annealing temperature and extension period using the following oligonucleotides as sense and antisense primers, respectively: 5'-GTATTTCTCATTCTTGGTAA-3', 5'-CTGGGGCAGGCGGGGCGCAGAA-3'; 5'-GGATTTGGTGGGCAAACTGC-3', 5'-CTGGGGCAGGCGGGGCGCAGAA-3'; 5'-CGCGAGCTCCCAGGTCCCAGAGATGAGAG-3', 5'-CGCAAGCTTCTGGGGCAGGCGGGGGCGCAC-3'; 5' -CGCGA- 
GCTCGGATTTGGTGGGCAAACTGCA-3', and CGCAAGCTTGACGCAAGGCAAAAGCGGCAG.

AhRR $(-633 /+71)$ and AhRR $(-269 /+71)$ were cloned into the blunt-ended SmaI site of a pGL3-Basic vector, whereas AhRR $(-20 /+71)$ and AhRR $(-269 /-86)$ were digested with SacI and HindIII and cloned into the cohesiveended pGL3-Basic vector digested with the same pair of restriction enzymes. Site-directed mutagenesis was performed on the AhRR (-269/+71)-pGL3-Basic plasmid using the QuickChange Site-directed Mutagenesis Kit (Stratagene, CA, U.S.A.). In this promoter region, there are two AhREs, AhRE1 and AhRE2, from the start site. The following two sets of forward and reverse primer pairs were employed to introduce missense mutations into AhRE1 and AhRE2 (the constructs were designated AhRR (-269/+71) AhRE1MUTpGL3-Basic and AhRR (-269/+71) AhRE2MUT-pGL3Basic, respectively): 5'-GGCAGGGCCGCTCTCTAGCTGGGGTGGGGC-3', 5'-GCCCCACCCCAGCTAGAGAGCGGCCCTGCC-3'; 5'-GGATCAGGATGTTCTAGCGCCCCCTGCCGC-3', 5' -GCGGCAGGGGGCGCTAGAACATCCTGATCC-3' (altered nucleotides are underlined).

The double mutant AhRR $(-269 /+71)$ AhRE1, 2MUTpGL3-Basic was constructed in a stepwise fashion. RL-34 cells were subcultured on 24-well plates. Twenty-four hours later, the cells were transfected in each well with $200 \mathrm{ng}$ of the respective AhRR-pGL3-Basic plasmid and $25 \mathrm{ng}$ of phRL-SV40 plasmid. In a negative control, the pGL3-Basic plasmid was employed instead of the AhRR-pGL3-Basic plasmid. Cells were cultured overnight and treated with either 3-MC $2.5 \mu \mathrm{M}$, dexamethasone (Dex) $100 \mathrm{~nm}$, or vehicle (DMSO) for the final $6 \mathrm{~h}$ before the reporter assay was performed. The final luminescence values were expressed as the ratio of firefly luciferase units to Renilla luciferase units.

RT-PCR Analysis of the bHLH/PAS Family Genes and CYP1A1 Total RNA was extracted from the homogenate of $90 \mathrm{mg}$ each of organs such as the liver, lung, kidney, heart, small intestine and cecum of male or female rats treated with $3-\mathrm{MC}$ in corn oil $(80 \mathrm{mg} / \mathrm{kg}$ body weight) using the RNeasy Mini Kit (Qiagen). The small intestinal tract was segmented longitudinally from the stomach to colon into three segments. The total RNA extract and oligo-dT primer were added to RTG You-Prime First-Strand Beads (Amersham Biosciences), and reverse transcription proceeded to obtain cDNA, to which the primer pair, Ex Taq polymerase (Takara, Japan), 10× Ex Taq Buffer, and dNTP mixture were added. After adjusting the total volume to $25 \mu \mathrm{l}$, the cDNA was amplified in a thermal cycler under the following conditions after preincubation at $95^{\circ} \mathrm{C}$ for $5 \mathrm{~min}$ : 30 cycles of denaturation at $98^{\circ} \mathrm{C}$ for $30 \mathrm{~s}$, annealing at $56^{\circ} \mathrm{C}$ for $30 \mathrm{~s}$, and extension at $72^{\circ} \mathrm{C}$ for $1 \mathrm{~min}$ (CYP1A1, AhR); 30 cycles of denaturation at $98^{\circ} \mathrm{C}$ for $30 \mathrm{~s}$, annealing at $60^{\circ} \mathrm{C}$ for $30 \mathrm{~s}$, and extension at $72{ }^{\circ} \mathrm{C}$ for $1 \mathrm{~min}$ (ARNT, ARNT2); 30 cycles of denaturation at $94^{\circ} \mathrm{C}$ for $45 \mathrm{~s}$, annealing at $60^{\circ} \mathrm{C}$ for $45 \mathrm{~s}$, and extension at $72^{\circ} \mathrm{C}$ for $1 \mathrm{~min}$ (AhRR); 25 cycles of denaturation at $98^{\circ} \mathrm{C}$ for $30 \mathrm{~s}$, annealing at $56^{\circ} \mathrm{C}$ for $30 \mathrm{~s}$, and extension at $72{ }^{\circ} \mathrm{C}$ for $1 \mathrm{~min}$ (glyceraldehyde-3-phosphate dehydrogenase: GAPDH). The following oligonucleotides were used as forward and reverse primers, respectively: $5^{\prime}$-GGCTTACCATGGGAACTGAG-3' and 5'-CCACCAGAGCAAAGCCATTGAG-3' for AhRR, 5'-TGGGGAGTTACTGGTCCTG-3' and 5'-CTGCCACTGGTTCACAAAGA-3' for
CYP1A, 5'-AAACAGTAAAGCCCGTCCCT-3' and 5'-AAGCTGGTCTGGCGTGTAGT-3' for AhR, 5'-TGTCATGTTCCGATTCCGAGC-3' and 5'-TAGTTGTCGGACCTAGCTGTG-3' for ARNT, 5'-TCACCTTCCAGAACCCCTACT3' and 5'-GGAGAAGATCGCATCTGCCTT-3' for ARNT2, and 5'-ACCACAGTCCATGCCATCAC-3' and 5'-TCCACCACCCTGTTGCTGTA-3' for GAPDH.

The reaction products were separated by agarose gel electrophoresis and analyzed using a Fluor Imager (Amersham Biosciences) after staining with ethidium bromide. The efficiency of reverse transcription was normalized by the band intensity for GAPDH cDNA.

Statistics Statistical difference was determined using Student's $t$-test. The 0.05 level of probability was adopted as significant.

\section{RESULTS AND DISCUSSION}

Nucleotide Sequence of AhRR mRNA and Structure of the AhRR Gene The full-length AhRR mRNA, which is 4694 bp long, including 79-bp 5'-UTR and 2509-bp 3'-UTR, and $2106 \mathrm{bp}$ coding seqence encoding a predicted protein of 701 amino acids, was sequenced in the heart. While this work was in progress, Korkalainen et al. ${ }^{3)}$ reported the $3^{\prime}$-end truncated sequence of rat AhRR mRNA of $2369 \mathrm{bp}$, including 79-bp 5'-UTR and 184-bp defective 3'-UTR. A homology search for rat AhRR cDNA using the BLAST2 program identified a rat genome sequence deposited with accession number AC131864. ${ }^{3)}$ The structure of rat AhRR genomic DNA consists of 11 exons and 10 introns, with the translation start site at the 9th nucleotide in the $73 \mathrm{bp}$ exon 2 and the stop site at $c a$. one-third of the 3500-bp exon 11 (Fig. 1). This structure of rat AhRR mRNA is similar to that for mice consisting of 88-bp 5'-UTR (about 94\% identical with the rat counterpart), 2106-bp coding sequence (about 94\%), and 2542-bp 3'-UTR (about 84\%) (Fig. 1B). On the other hand, the human AhRR mRNA consists of 56-bp 5'-UTR and 276bp 5'-UTR, which is remarkably short in comparison with the noncoding sequences of AhRRs for rats and mice, although the 2148-bp coding sequence (715 a.a.) was about $69 \%$ identical with the rat counterpart (Fig. 1C). If the threedimensional architecture was formed upstream from the polyA tail, for which the elongated 3'-UTR of mouse and rat AhRR mRNAs might be responsible, the longevity of mRNA would be extended due to steric hindrance. The bHLH and PAS A domains were highly conserved $(76.8 \%$ and $51.5 \%$ identical, respectively) between rat AhR and AhRR mRNAs, whereas PAS B and Q-rich domains are missing in AhRR. It is likely that AhRR lacks the binding capacity to HSP90 and ligand as well as transcriptional activation potential. The high homology of bHLH and PAS domains was maintained among rat, mouse, and human AhRRs (87-99\% and 99$100 \%$, respectively).

Quantification of AhRR, AhR, and CYP1A1 Expression in Various Organs of Male and Female Rats The expression of AhRR mRNA was semiquantitatively analyzed using RT-PCR in various organs such as the heart, lung, liver, kidney, and small intestinal fragments referred to as 1 through 3 longitudinally from the stomach to the colon, and cecum in the both presence and absence of 3-MC.

Although AhRR mRNA was expressed ubiquitously in all 
(A)

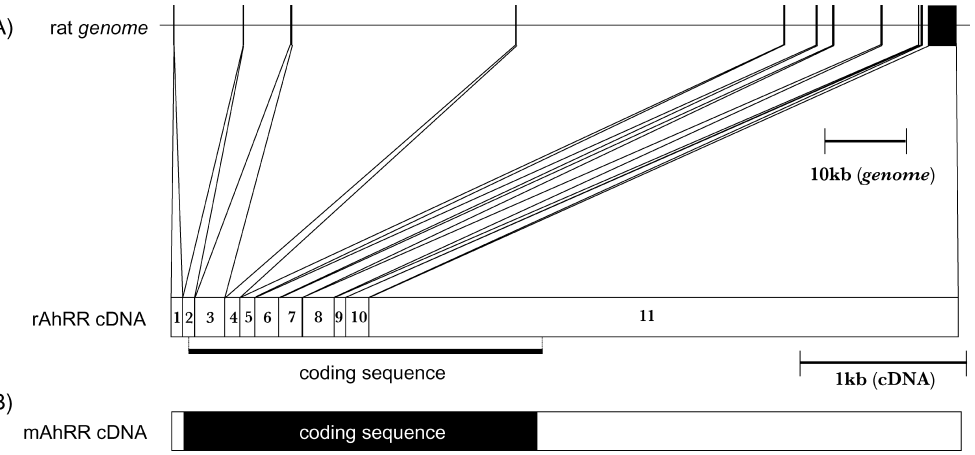

(C)

hAhRR cDNA $\quad$ coding sequence

Fig. 1. Structural Organization of the Rat Ahrr Gene and cDNA

For the rAhRR genomic sequence (AC131864), solid boxes represent exons. For the cDNA sequence (AB174900), the coding region is shown by an underlining solid bar and the number in each box indicates the exon number counted from the $5^{\prime}$-terminus. For the cDNA sequences of mouse and human AhRRs (AB015140 and NM020731, respectively), the coding regions are indicated by solid boxes and the $5^{\prime}$ - and $3^{\prime}$-UTRs are shown by open boxes.

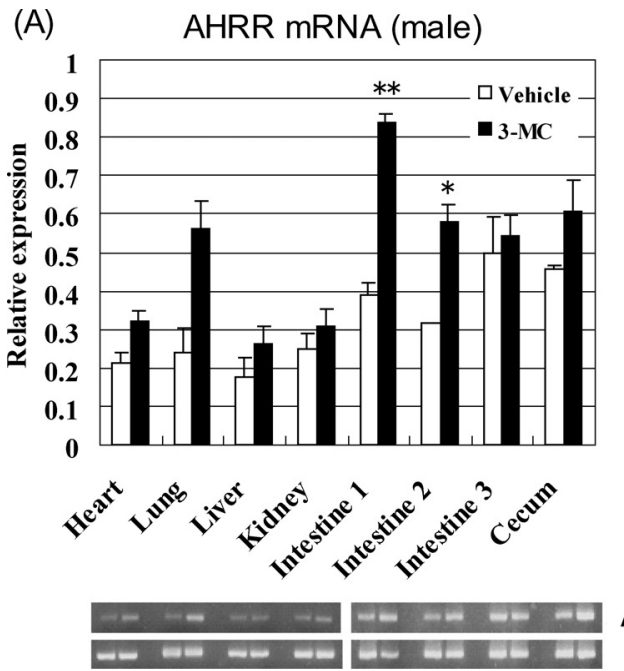

(B)
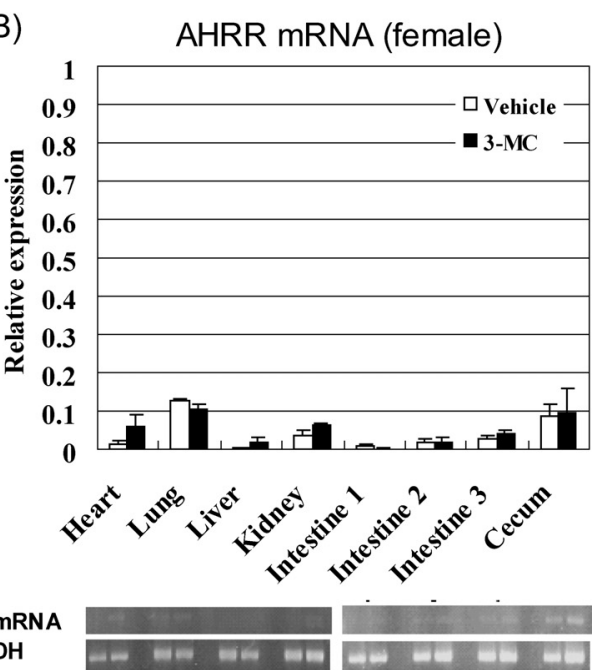

Fig. 2. Gender Difference in the Organ-Specific Constitutive and 3-MC-Inducibile Expression of AhRR mRNA

The expression of AhRR mRNA was measured semiquantitatively using RT-PCR in male (A) and female (B) Wistar rats (7 weeks old) $24 \mathrm{~h}$ after treatment with 3 -MC ( $80 \mathrm{mg} / \mathrm{kg}$ body weight) or corn oil vehicle (control). Duodenal, jejunal, and distal ileal segments were referred to as intestine 1,2 , and 3 , respectively. The efficiency of reverse transcription was normalized by the band intensity of GAPDH cDNA. Data are expressed as mean \pm S.E. $(n=3)$ with significant differences compared with individual vehicle controls at $* p<0.05$ and $* * p<0.01$.

organs tested as well as AhR mRNA, it was relative high in the small intestine and cecum in a male-dominant sexual dimorphic fashion, reflecting gender differences in the expression of AhR mRNA (Figs. 2, 3). In untreated mice, essentially no expression of AhRR mRNA was detected in the liver, heart, lung, kidney, intestine, and thymus. ${ }^{1)}$ Upon treatment with 3-MC, however, AhRR mRNA levels were induced in these tissues, although the heart and lung showed the most abundant AhRR mRNA expression, whereas the liver, thymus, kidney, and intestine expressed relatively small amounts of mRNA. The levels of human AhRR expression were extremely high in the testis, very high in the lung, ovary, spleen, and pancreas, and poor or negligible in the small intestine, liver, kidney, colon, and heart. ${ }^{14)}$ In summary, the organ-specific expression profiles of AhRR mRNA differ markedly among species.

The constitutive expression of CYP1A1 showed similar organ specificity except for the low expression in the cecum (Fig. 4). Bilirubin secreted into bile is considered an endoge- nous ligand of AhR. ${ }^{15)}$ Therefore the high level of expression of two AhR-target genes, AhRR and CYP1A1, in the intestinal fragments could be attributed to the chronic exposure of epithelial cells in the small intestine to unconjugated bilirubin. In this case, the negative effect of AhRR on the expression of CYP1A1 was not significant. In the presence of an extra exogenous ligand, i.e., 3-MC, the transcriptional induction of AhRR continued in the small intestine, especially in the region proximal to the stomach, while the expression of CYP1A1 was effectively inhibited by the increased level of AhRR. The insensitivity of the hepatic expression of the AhRR transcript to induction by the AhR agonist 3-MC is rational because the liver, having many metabolic enzymes represented by CYP1A1, plays a pivotal role in the detoxification of drugs and other xenobiotics.

Quantification of ARNT and ARNT2 Expression in Various Organs of Male and Female Rats As can be seen in Figs. 2 and 4, the organ-specific expression profiles of constitutive AhRR and CYP1A1 mRNAs were not synchro- 
(A)
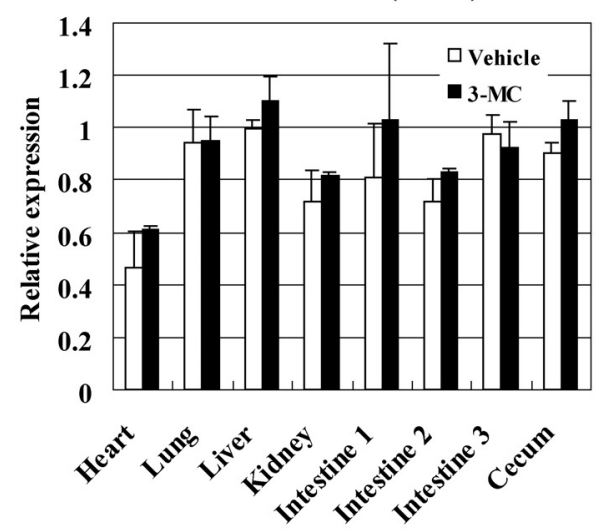

(B)

AhR mRNA (female)

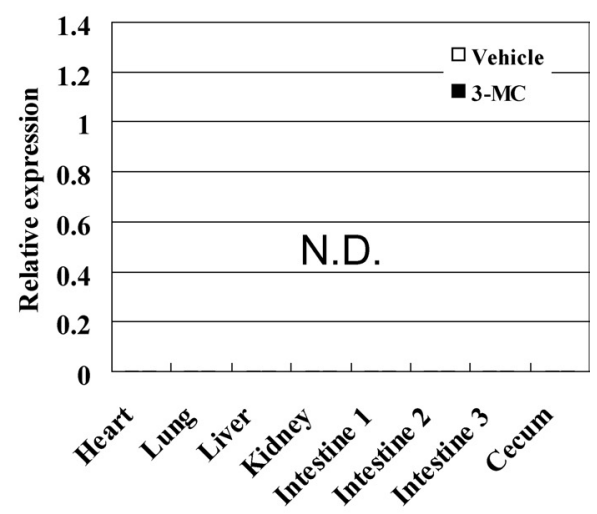

AhR mRNA

GAPDH

Fig. 3. Gender Differences in the Organ-Specific Constitutive and 3-MC-Inducibile Expression of AhR mRNA

The expression of AhR mRNA was measured semiquantitatively using RT-PCR in male (A) and female (B) Wistar rats (7 weeks old) treated as described in the legend to Fig. 2. Data are expressed as mean \pm S.E. $(n=3)$.

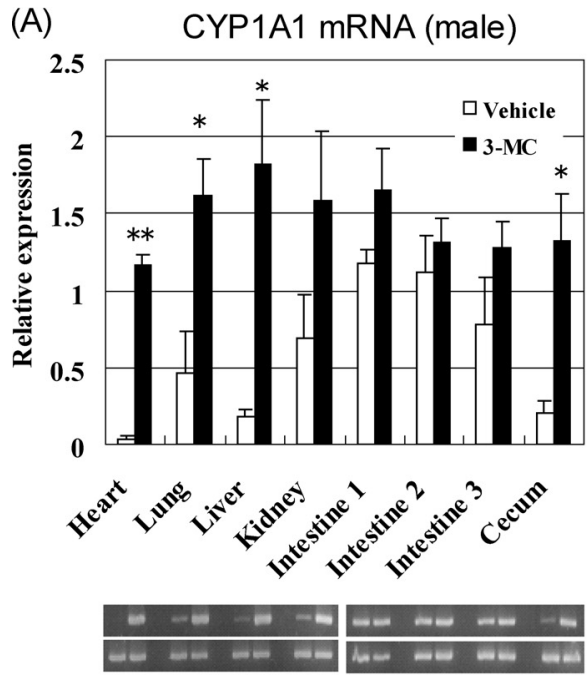

(B) CYP1A1 mRNA (female)

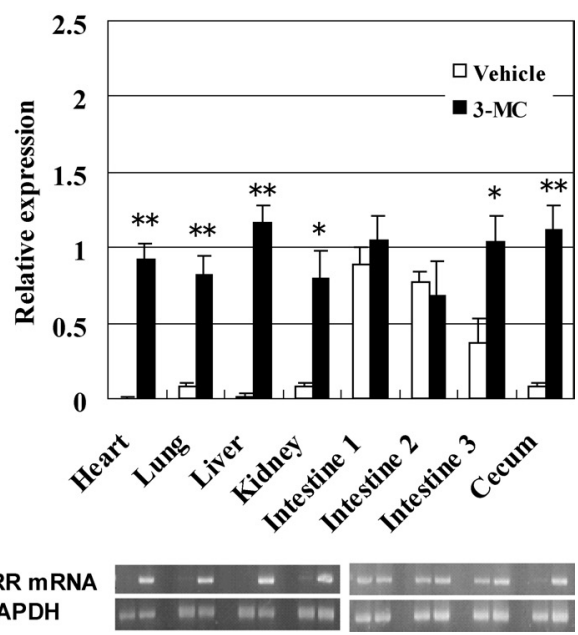

Fig. 4. Gender Differences in the Organ-Specific Constitutive and 3-MC-Inducibile Expression of CYP1A1 mRNA

The expression of CYP1A1 mRNA was measured semiquantitatively using RT-PCR in male (A) and female (B) Wistar rats (7 weeks old) treated as described in the legend to Fig. 2. Data are expressed as mean \pm S.E. $(n=3)$ with significant differences compared to individual vehicle controls at $* p<0.05$ and $* * p<0.01$.

nized, although both are target genes of AhR. Shuttling between nuclear and cytoplasmic components even in the absence of exogenous ligands, AhR may contribute to the constitutive expression of target genes. AhR functions as a ligand-activated transcription factor forming a heterodimer complex with ubiquitous ARNT or its homologue preferentially expressed in the brain and kidney, ARNT2. ${ }^{16}$ ) Furthermore, they are dimerization partners not only for AhR but also for several other members of the bHLH/PAS family, playing a central role in regulating divergent signaling pathways. ${ }^{17)}$ In addition to the organ distribution profile, the partner preference distinguishes ARNT from ARNT2. The expression of ARNT2 mRNA was exclusively observed in the lung and kidney, where the high level of constitutive expression of CYP1A1 mRNAs was marked, but not in the liver and heart (Fig. 5), implying a pivotal role of ARNT2 in the constitutive expression of CYP1A1 mRNA.

Effects of AhRR on AhR-Dependent Transcriptional
Activation in RL-34 Cells The rat liver-derived RL-34 cells were transiently transfected with the AhRR expression plasmid in the range of 0 to $0.20 \mu \mathrm{g} /$ well together with a fixed amount $(0.20 \mu \mathrm{g} /$ well $)$ of reporter plasmid preceded by the AhRE enhancer sequence. After total DNA was adjusted to $0.40 \mu \mathrm{g} /$ well using an empty plasmid, the cells were cultured in the presence and absence of 3-MC. The dose-dependent inhibition of AhR-dependent transcriptional activation of the reporter gene was shown by AhRR (Fig. 6). Like AhRRs of human, mouse, and fish origin, the rat orthologue was confirmed to inhibit the constitutive and inducible transactivation of the AhR target gene.

Structural and Functional Analysis of the Rat AhRR Promoter Motif analysis was conducted on the promoter region up to $1221 \mathrm{bp}$ upstream from the AhRR gene (Fig. 7). For the functional analysis of these motifs, PCR amplicons of various upstream regions were cloned into a pGL3Basic vector (Fig. 8). On transfection into RL-34 cells, the 
(A)

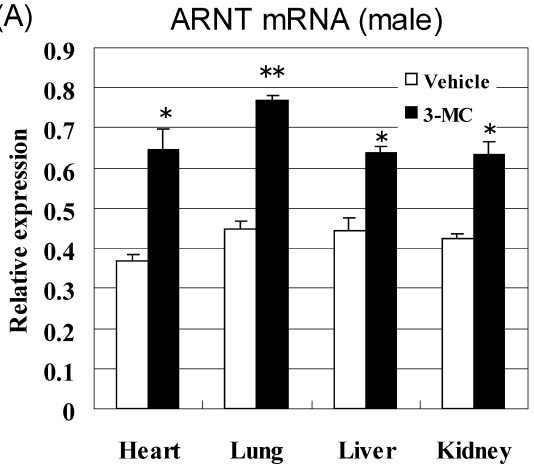

(B)

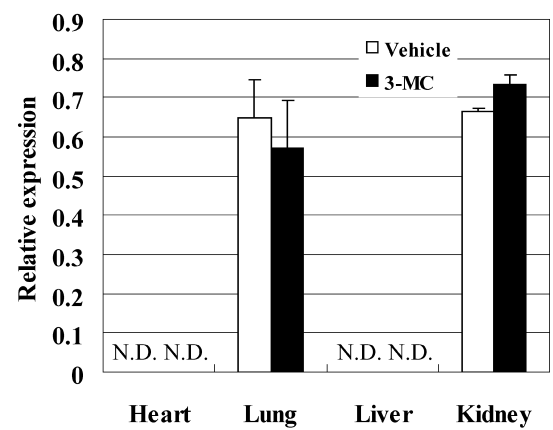

Fig. 5. Organ-Specific Constitutive and 3-MC-Inducibile Expression of ARNT and ARNT2 mRNA

The expression of ARNT (A) and ARNT2 (B) mRNA was measured semi-quantitatively using RT-PCR in male Wistar rats (7 weeks old) treated as described in the legend to Fig. 2. Data are expressed as mean \pm S.E. $(n=3)$ with significant differences compared with individual vehicle controls at $* p<0.05$ and $* * p<0.01$.

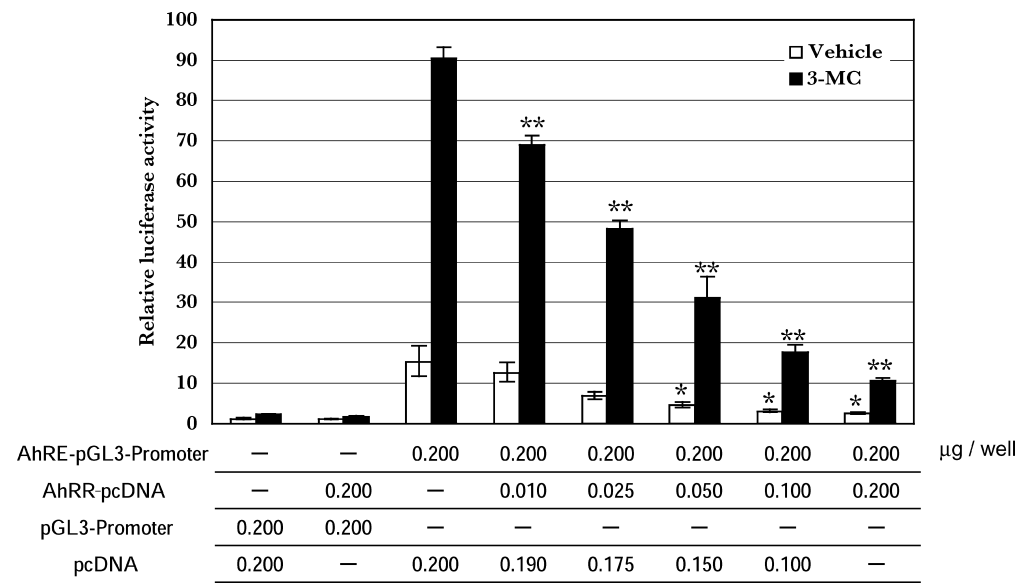

Fig. 6. Inhibition of Rat AhR-Dependent Transcriptional Activation by rAhRR

RL-34 cells were cotransfected with divergent amounts of the AhRR-pcDNA construct and a fixed amount of AhRE-pGL3-Promoter plasmid, in which the expression of the luciferase gene was under the control of an artificial enhancer consisting of four AhRE repeats, both in the presence and absence of 3-MC. Data are normalized by luciferase activity in cells transfected with pGL3-Promoter vector in the absence of 3-MC and expressed as mean \pm S.E. $(n=3)$ with significant differences compared with cells transfected with AhRE-pGL3-Promoter not accompanied by AhRR-pcDNA either in the presence or absence of 3-MC at $* p<0.05$ and $* * p<0.01$.

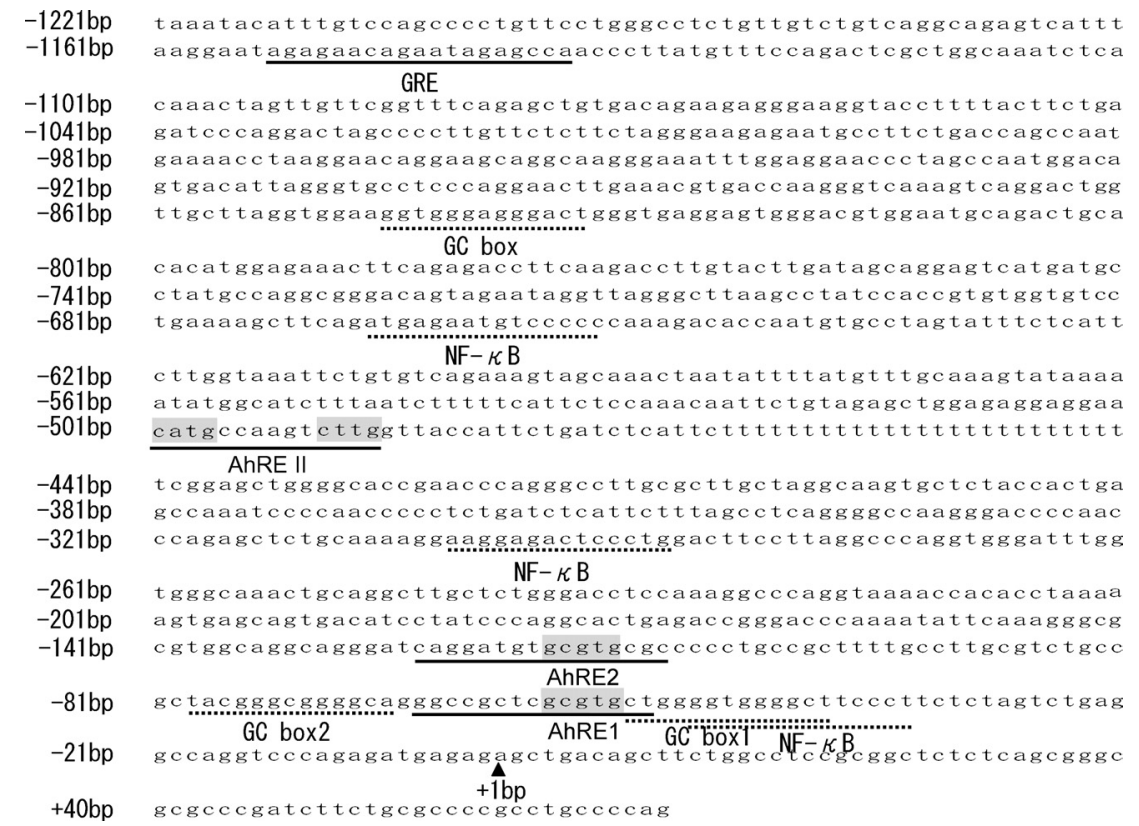

Fig. 7. Sequence of the $5^{\prime}$-Upstream Region of the rAhRR Gene

The positions are numbered from the transcription start site indicated by the arrowhead. Putative regulatory elements are indicated, and AhRE and GC box sequences are numbered upstream from the transcription start site. The AhRE invariant core sequence (GCGTG) and proposed AhRE II sequence (CATGN6CA/TTG) in the human CYP1A2 gene are shaded. 
highest luciferase activity was observed with pGL3-AhRR $(-269 /+71)$ in the absence of 3-MC. As cells transfected with either pGL3-AhRR $(-20 /+71)$ or pGL3-AhRR $(-269 /-86)$ showed the same level of luciferase activity as those transfected with the pGL3-Basic vector, the response element for the basal transcription complex could be allocated between -86 and -20 . As shown in Fig. 7, the existence of double GC boxes, double AhREs (AhRE1 and 2), and single $\mathrm{NF}-\kappa \mathrm{B}$ site were deduced by motif analysis. GC boxes, as seen in the vicinity of the transcriptional start site

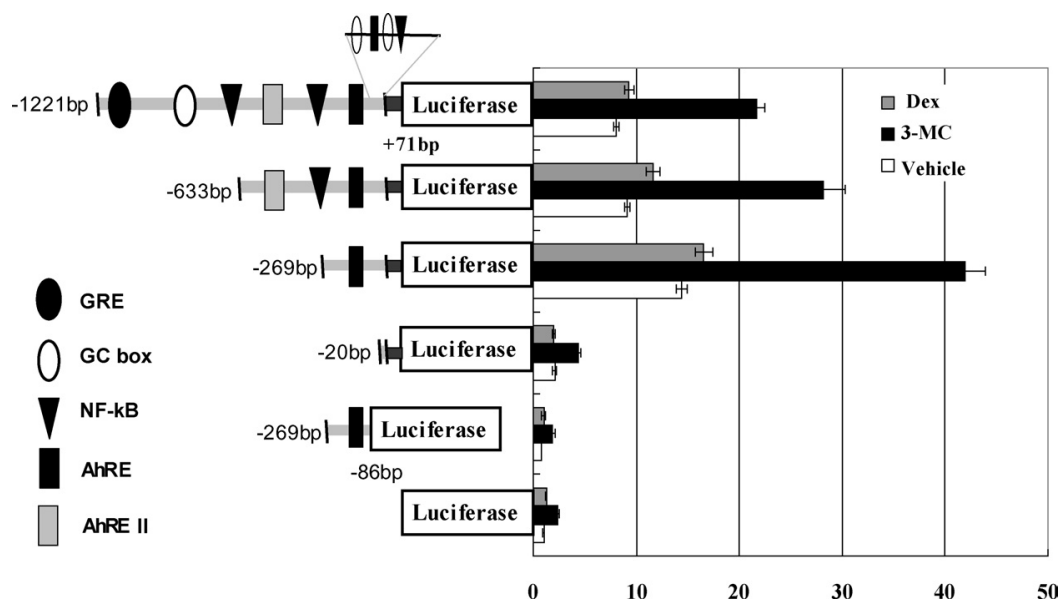

Fig. 8. Deletion Analysis of the Putative Functional Domains in the rAhRR Promoter

The pGL3-Basic vector inserted with each differentially truncated promoter sequence of the rAhRR gene was constructed. RL-34 cells were transfected with individual reporter plasmids simultaneously with phRL-SV40, which was used as an internal standard. Luciferase activity was measured in the presence of either 3-MC or Dex. The vehicle (DMSO) control was run in parallel. Top lane, AhRR (-1221/+71)-pGL3-Basic; 2nd lane, AhRR (-633/+71)-pGL3-Basic; 3rd lane, AhRR (-269/+71)-pGL3-Basic; 4th lane, AhRR $(-20 /+71)$-pGL3-Basic; 5th lane, AhRR $(-269 /-86)$-pGL3-Basic; and bottom lane, pGL3-Basic vector. The luciferase activity of each reporter construct is presented as a relative value (-fold) to the pGL3-Basic vector. Data are mean \pm S.E. $(n=3)$.

(A)

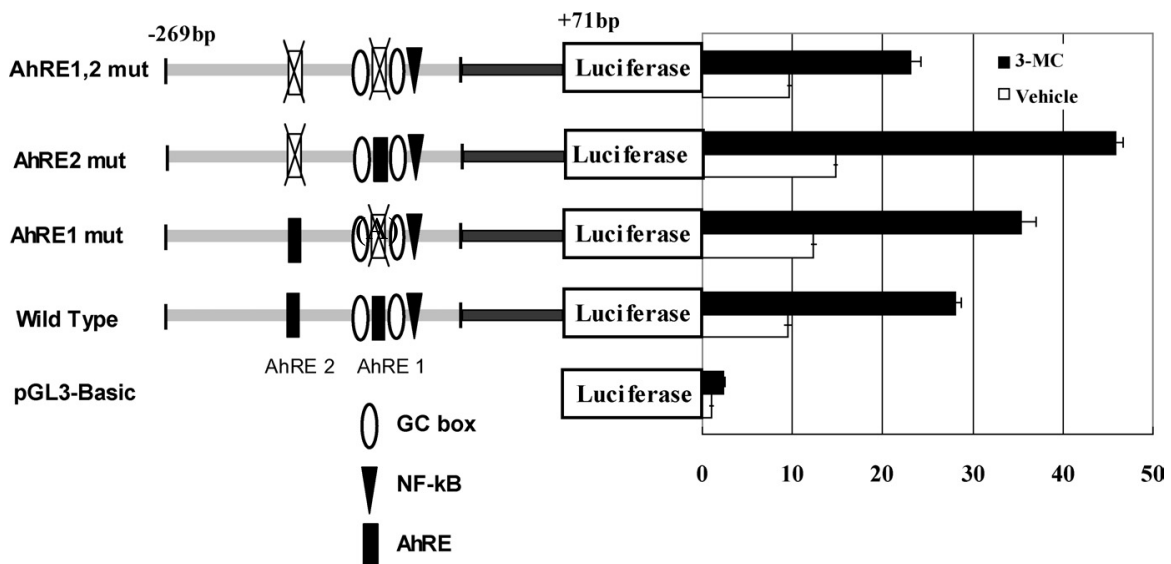

(B)

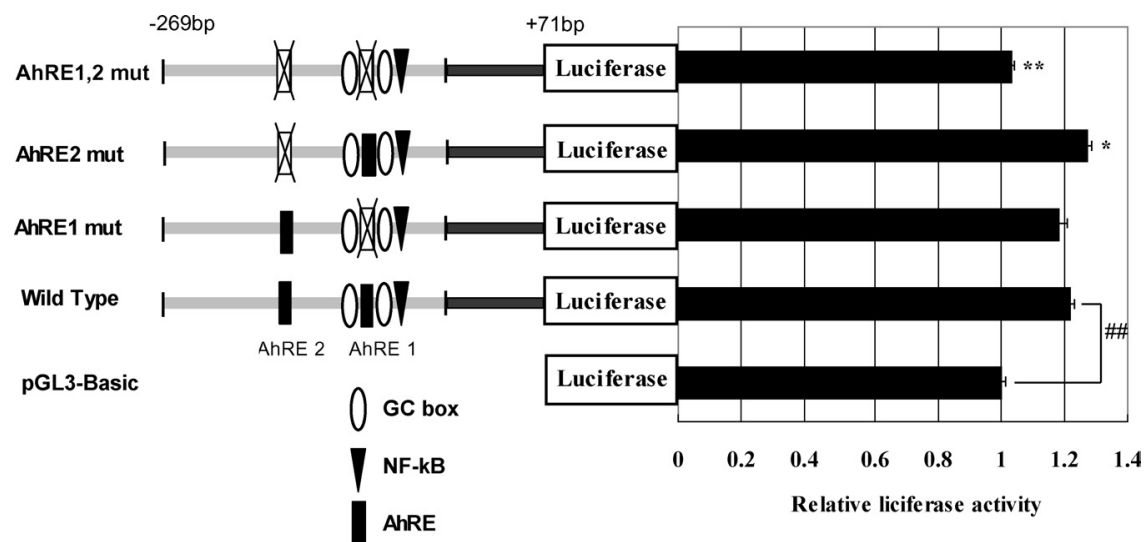

Fig. 9. Mutational Analysis for the Role of AhREs in the AhRR Promoter

The missense mutation was introduced into the AhRE1 and/or AhRE2 domains of AhRR ( $-269 /+71)$-pGL3-Basic. RL-34 cells were transfected with wild-type or individual mutant reporter constructs simultaneously with phRL-SV40. (A) Luciferase activity was measured in the presence of 3-MC. The vehicle control was run in parallel. Top lane, AhRR (-269/+71)-AhRE1,2mut-pGL3-Basic; 2nd lane, AhRR (-269/+71) AhRE2mut-pGL3-Basic; 3rd lane, AhRR (-269/+71) AhRE1mut-pGL3-Basic; 4th lane, AhRR $(-269 /+71)$-pGL3-Basic (wild type); and 5th lane, pGL3-Basic vector. (B) Luciferase activity in the presence of 3-MC with each reporter plasmid divided by the corresponding vehicle control value is presented. Data represent mean \pm S.E. $(n=3)$ with significant differences compared with the wild type at $* p<0.05, * * p<0.01$ and $\# p<0.01$. 
of the TATA-less AhRR gene, are known to be binding sites for SP1 family transcriptional factors, which in turn might cooperate with AhR/ARNT heterodimers retained on the AhREs, synergistically activating the transcription of target genes. ${ }^{18)}$ The expression levels of the reporter gene by cells transfected with plasmids harboring the promoter regions elongated to different extents toward the upstream region, such as pGL3-AhRR $(-633 /+71)$ and pGL3-AhRR $(-1221 /+71)$ were lower than that for pGL3-AhRR $(-269 /+71)$, implying the existence of a silencer(s) between -1221 and $-269 \mathrm{bp}$.

As for the 3-MC-mediated induction of luciferase activity, however, even cells transfected with pGL3-Basic vector showed a 2.2-fold increase. Since this unexpected phenomenon was not observed with the glucocorticoid receptor agonist Dex, and the agonists of xenobiotic sensor nuclear receptors, PXR and CAR (data not shown), it might be attributable to putative AhREs residing on this naive plasmid. Having putative endogenous AhREs, the pGL3-Basic vector itself showed a positive response to the 3-MC-dependent induction of the reporter gene (Fig. 8). Plasmids such as pGL3-AhRR $(-269 /+71)$, pGL3-AhRR $(-633 /+71)$, and pGL3-AhRR $(-1221 /+71)$ afforded the transfected cells with significantly enhanced values when compared with the pGL3-Basic vector. AhRE II at around $-500 \mathrm{bp}$, on which the AhRARNT heterodimer works as a coactivator unlike classical AhRE, ${ }^{19)}$ is widely distributed in various 3-MC-responsive genes, and the functional integrity of this cis-element was indicated by the highest value obtained with pGL3-AhRR $(-633 /+71)$. However, the putative GRE assigned in the vicinity of $-1150 \mathrm{bp}$ was found to be transcriptionally inert.

To evaluatie the respective contributions of two AhRE1 and AhRE2 to constitutive and 3-MC-inducible luciferase activity independently from the effects of AhRE II, RL-34 cells were transfected with pGL3-AhRR $(-269 /+71)$ with missense mutations in AhRE1 and/or AhRE2. Although AhRE1 and AhRE2 are active individually, they adversely interfere with each other in terms of both constitutive and inducible expressions of the reporter gene (Fig. 9). Based on these findings, AhREs in the vicinity of the transcriptional start point of the AhRR gene are responsible for the moderate response of rat AhRR to AhR agonists represented by 3-MC.

Finally, epidemiologic studies have just started recently on the polymorphism of human AhRR in terms of CYP1A1 inducibiliity, lung cancer, and male infertility, ${ }^{20-22)}$ while no difference in the structure and expression profile of AhRRs was observed between TCDD-sensitive and -resistant rat strains. $^{3)}$

\section{REFERENCES}

1) Mimura J., Ema M., Sogawa K., Fujii-Kuriyama Y., Genes Dev., 13, $20-25$ (1999).

2) Fukunaga B. N., Probs M. R., Reisz-Porszasz S., Hankinson O., J. Biol. Chem., 270, 29270-29278 (1995).

3) Korkalainen M., Tuomisto J., Pohjanvirta R., Biochem. Biophys. Res. Commun., 315, 123-131 (2004).

4) Kazlauskas A., Poellinger L., Pongratz I., J. Biol. Chem., 275, 41317-41324 (2000).

5) Watanabe T., Imoto I., Kosugi Y., Fukuda Y., Mimura J., Fujii Y., Isaka K., Takayama M., Sato A., Inazawa J., J. Hum. Genet., 46, 342-346 (2001).

6) Karchner S. I., Franks D. G., Powell W. H., Hahn M. E., J. Biol. Chem., 277, 6949-6959 (2002).

7) Evans B. R., Karchner S. I., Franks D. G., Hahn M. E., Arch. Biochem. Biophys., 441, 151-167 (2005).

8) Baba T., Mimura J., Gradin K., Kuroiwa A., Watanabe T., Matsuda Y., Inazawa J., Sogawa K., Fujii-Kuriyama Y., J. Biol. Chem., 276, $33101-33110$ (2001).

9) Whitlock J. P., Jr., Annu. Rev. Pharmacol. Toxicol., 39, 103-125 (1999).

10) Korkalainen M., Tuomisto J., Pohjanvirta R., Biochem. Biophys. Res. Commun., 303, 1095-1100 (2003).

11) Nagase T., Ishikawa K., Kikuno R., Hirosawa M., Nomura N., Ohara O., DNA Res., 29, 337-345 (1999).

12) Shimba S., Wada T., Tezuka M., J. Cell Sci., 114, 2809-2817 (2001).

13) Shimba S., Komiyama K., Moro I., Tezuka M., J. Biochem. (Tokyo), 132, 795-802 (2002).

14) Yamamoto J., Ihara K., Nakayama H., Hikino S., Satoh K., Kubo N., Iida T., Fujii Y., Hara T., Life Sci., 74, 1039-1049 (2004).

15) Sinal C. J., Bend J. R., Mol. Pharmacol., 52, 590_599 (1997).

16) Hosoya T., Oda Y., Takahashi S., Morita M., Kawauchi S., Ema M., Yamamoto M., Fujii-Kuriyama Y., Genes Cells, 6, 361-374 (2001).

17) Kewley R. J., Whitelaw M. L., Chapman-Smith A., Int. J. Biochem. Cell Biol., 36, 189-204 (2004).

18) Kobayashi A., Sogawa K., Fujii-Kuriyama Y., J. Biol. Chem., 271 12310-12316 (1996).

19) Sogawa K., Numayama-Tsuruta K., Takahashi T., Matsushita N., Miura C., Nikawa J., Gotoh O., Kikuchi Y., Fujii-Kuriyama Y., Biochem. Biophys. Res. Commun., 318, 746-755 (2004).

20) Fujita H., Kosaki R., Yoshihashi H., Ogata T., Tomita M., Hasegawa T., Takahashi T., Matsuo N., Kosaki K., Teratology, 65, 10-18 (2002).

21) Cauchi S., Stuecker I., Cenee S., Kremers P., Beaune P., Massaad-Massade L., Pharmacogenetics, 13, 339-347 (2003).

22) Watanabe M., Sueoka K., Sasagawa I., Nakabayashi A., Yoshimura Y., Ogata T., Fertil. Steril., 82, 1067-1071 (2004). 